Systematic Review/Meta-Analysis

\title{
Proximal Hamstring Tendinopathy: A Systematic Review of Interventions
}

\author{
Anthony Michael Nasser, $\mathrm{PT}^{1}$ a , Bill Vicenzino, $\mathrm{PT}, \mathrm{PhD}^{2}$, Alison Grimaldi, $\mathrm{PT}, \mathrm{PhD}^{2}$, Jay Anderson, $\mathrm{PT}^{3}, \mathrm{Adam}_{\mathrm{Ivan}}$ \\ Semciw, PT, PhD ${ }^{4}$ \\ 1 Department of Rehabilitation, Nutrition and Sport, La Trobe University; Graduate School of Health, University of Technology Sydney, 2 School of \\ Health and Rehabilitation Sciences, University of Queensland, ${ }^{3}$ Gold Coast Suns Football Club, ${ }^{4}$ Department of Rehabilitation, Nutrition and Sport, La \\ Trobe University; Northern Centre for Health Education and Research, Northern Health \\ Keywords: tendinopathy, surgery, hamstring tendon, buttock pain \\ $10.26603 / 001$ c. 21250
}

International Journal of Sports Physical Therapy

\section{Background}

Proximal hamstring tendinopathy affects athletic and non-athletic populations and is associated with longstanding buttock pain. The condition is common in track and field, long distance running and field-based sports. Management options need to be evaluated to direct appropriate clinical management.

\section{Purpose/Hypothesis}

To evaluate surgical and non-surgical interventions used in managing proximal hamstring tendinopathy.

\section{Study design}

Systematic review

\section{Methods}

Electronic databases were searched to January 2019. Studies (all designs) investigating interventions for people with proximal hamstring tendinopathy were eligible. Outcomes included symptoms, physical function, quality of life and adverse events. Studies were screened for risk of bias. Reporting quality was assessed using the Cochrane Risk of Bias Tool (Randomized Controlled Trials [RCT]) and the Joanna Briggs Institute Checklist (Case Series). Effect sizes (Standard mean difference or Standard paired difference) of 0.2, 0.5 and 0.8 were considered as small, medium and large respectively. Overall quality of evidence was rated according to GRADE guidelines.

\section{Results}

Twelve studies (2 RCTs and 10 case series) were included ( $\mathrm{n}=424$; males 229). RCTs examined the following interventions: platelet-rich plasma injection $(n=1)$, autologous whole-blood injection $(n=1)$, shockwave therapy $(n=1)$ and multi-modal intervention $(n=1)$. Case series included evaluation of the following interventions: platelet-rich plasma injection $(n=3)$, surgery $(n=4)$, corticosteroid injection $(n=2)$, multi-modal intervention + platelet-rich plasma injection $(n=1)$. Very low-level evidence found shockwave therapy was more effective than a multi-modal intervention, by a large effect on improving symptoms (-3.22 SMD; 95\% CI -4.28, -2.16) and physical function (-2.42 SMD; 95\% CI-3.33, -1.50) in the long-term. There was very low-level evidence of no difference between autologous whole-blood injection and platelet-rich plasma injection on physical function $(0.17$ SMD; $95 \%$ CI $-0.86,1.21)$ to $(0.24$ SMD; $95 \%$ CI $-0.76,1.24)$ and quality of life $(-0.04$ SMD; $95 \%$ CI $-1.05,0.97)$ in the medium-term. There was very low-quality evidence that surgery resulted in a large reduction in symptoms (-1.89 SPD; 95\% CI -2.36, $-1.41)$ to (-6.02 SPD; $95 \%$ CI $-8.10,-3.94)$ and physical function (-4.08 SPD; 95\%CI -5.53, $-2.63)$ in the long-term.

\footnotetext{
a Corresponding Author: Anthony Nasser La Trobe University, Department of Rehabilitation, Nutrition and Sport Melbourne, Victoria, Australia a.nasser@latrobe.edu.au M: 610295147337
} 


\section{Conclusions}

There is insufficient evidence to recommend any one intervention over another. A pragmatic approach would be to initially trial approaches proven successful in other tendinopathies.

\section{Level of evidence}

Level $2 \mathrm{a}$

\section{INTRODUCTION}

Proximal hamstring tendinopathy presents as persistent buttock pain that occurs with activities such as running, sitting and lunging. ${ }^{1,2}$ The condition primarily affects active men and women ${ }^{3}$ in sports such as track and field, distance running, soccer and rugby. ${ }^{1}$ It also afflicts people who do not participate in sport. ${ }^{4,5}$ The condition is challenging to manage because of the persistence of symptoms and lack of response to treatment. 3,6

Interventions for proximal hamstring tendinopathy focus on reducing symptoms and restoring physical function. Common non-surgical interventions include exercise, corticosteroid injection, platelet-rich-plasma injection and shockwave therapy. ${ }^{6,7}$ Common surgical procedures include tenotomy, bursal and tendon debridement and removal of adhesions around the sciatic nerve. ${ }^{2,8}$

With many treatment options available, a rational starting point is to systematically review the literature and synthesize information where possible. The purpose of this systematic review was to evaluate both surgical and nonsurgical interventions used in managing proximal hamstring tendinopathy.

\section{METHODS}

The systematic review protocol (Figure 1) was developed in accordance with the PRISMA statement and preregistered on PROSPERO (ID: CRD42017072678). ${ }^{9}$

A systematic literature search was conducted from the earliest date available until January 2019 for relevant studies published in MEDLINE, CINAHL, EMBASE, SPORTSDISCUS and PUBMED. A comprehensive search was undertaken, with assistance of a librarian, using a combination of keywords and medical subject headings (MeSH). The search strategy was formed around two topics; "hamstring" and "tendinopathy". Synonyms within each concept were combined with OR Boolean operator; and terms between concepts were combined with AND Boolean operator (Appendix 1).

After removal of duplicates, two reviewers (AN and JA) independently scanned titles and abstracts of all papers. Any disagreement was referred to a third reviewer (AS) for consensus. Full text versions of articles were obtained for all remaining studies. To ensure all relevant articles were included, citation tracking (PubMed/ Google Scholar) and reference checking of included studies was performed.

Study designs included were randomized controlled trials (RCTs), prospective (comparative) cohort studies, casecontrol studies and case series with 10 or more participants. Articles were confined to English language only. Biomechanical reports and narrative reviews were excluded. Par- ticipants of any age diagnosed with proximal hamstring tendinopathy by a health professional were included. Synonyms considered included: hamstring tendinitis, highhamstring tendinopathy, and hamstring origin tendinopathy. Traumatic injuries such acute proximal hamstring tendon tears, complete hamstring tears or avulsion injuries were excluded.

Surgical and non-surgical interventions were considered in this review. Interventions included, but were not limited to: surgery, shockwave therapy, platelet-rich plasma injections, autologous whole blood injections, corticosteroid injection and multi-modal intervention (NSAIDs, manual therapy, exercise and stretching).

Outcome measures that reported on symptoms, physical function (e.g. return to sport), quality of life (QOL) and ratings of success were included. Short ( $\leqslant 12$ weeks), medium (> 12 weeks -1 year) and long ( $\geqslant 1$ year) time-points were considered. Adverse events were reported as a secondary outcome, and were dichotomised as minor or major. ${ }^{10}$ Minor adverse events were defined as incidents which had minimal serious or potentially serious effects, such as thigh paraesthesia or a small infection that resolved with anti-microbial drugs. Major adverse events were incidents that had the potential for severe effects, such as deep vein thrombosis or severe infection.

One amendment was made from the initial protocol. Originally only case series were included. This was amended to exclude case series with $<10$ participants. This was identified as an oversight in the initial protocol and was revised prior to conducting the search. This amendment resulted in the exclusion of three case series with small sample sizes. ${ }^{11-13}$

Pre-specified data was extracted from each study and included eligibility criteria, study design, participant demographics, intervention, outcome parameters, results at all time points and adverse events. Data was extracted by one reviewer (AN) and checked by a second reviewer (AS). Authors were contacted in the case of missing data. If author(s) did not respond, they were contacted again after two weeks. If the author(s) still did not respond raw data was reported.

Means and standard deviations of continuous outcomes for comparative studies (e.g. intervention A vs Intervention B) were converted to standardized mean differences (SMD) with 95\%CI using RevMan (version 5.3). When studies reported changes over time, means and standard deviations (SD) were converted to standardized paired differences (SPD) using the 'metafor' package (version 2.1-0) within the ' $R$ ' statistical software package (version 3.5.1). SPD calculations (with 95\% CI) require information about pre- and post-test reliability. ${ }^{14,15}$ If this was not reported, or could not be located, a conservative estimate was used ( $\mathrm{r}=0.50)$. In cases where the SD was not reported, we used the for- 


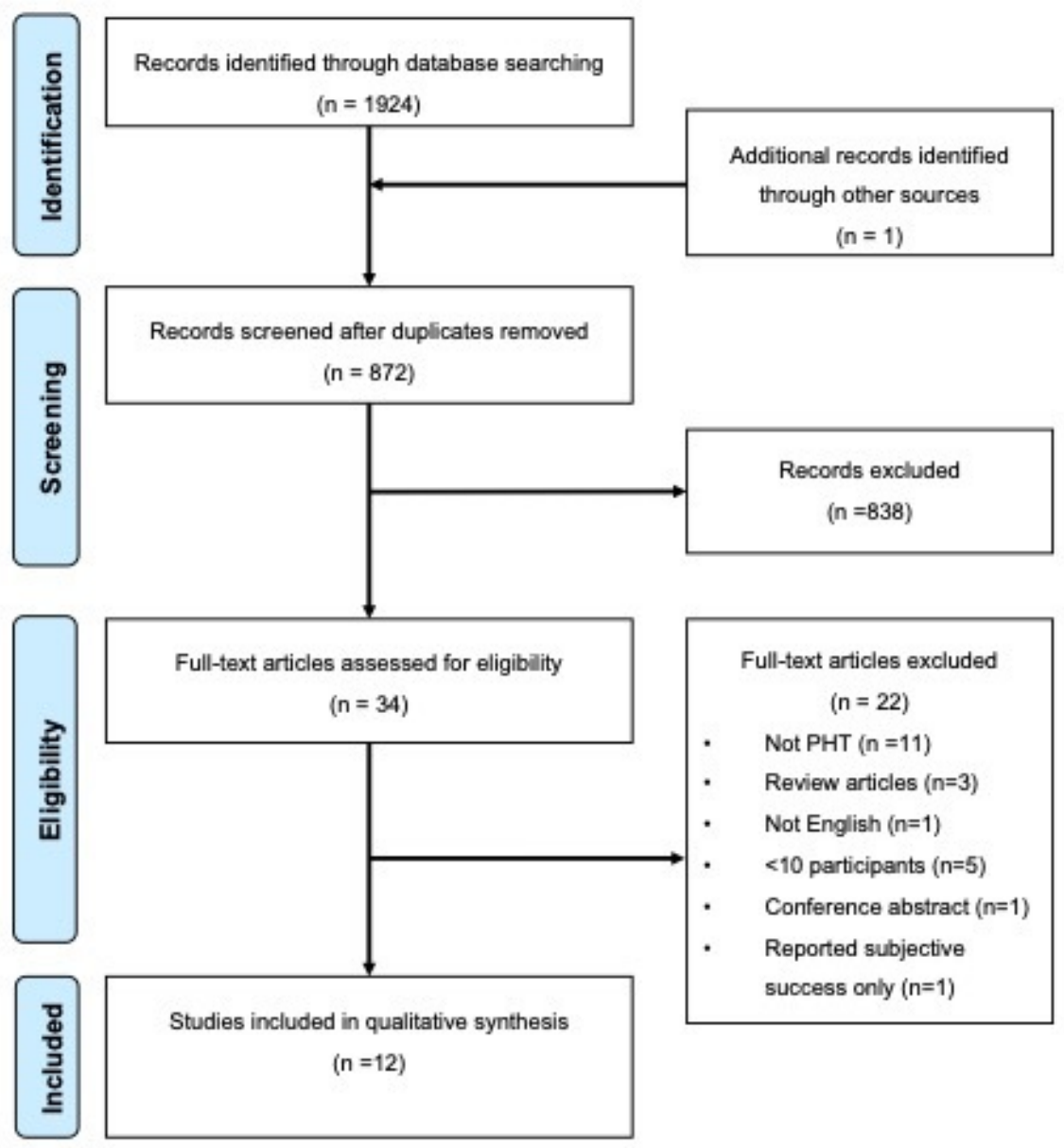

Figure 1: PRISMA flow chart illustrating study selection process

mula provided in the Cochrane Handbook (section 16.1.3.2), using a conservative correlation coefficient of 0.5 , to calculate the SD. ${ }^{16}$ Effect sizes (SMDs and SPDs) of 0.2, 0.5 and 0.8 were considered as small, medium and large respectively. ${ }^{17}$ When data could not be presented as a SPD, raw data were presented. The proportion of people who returned to pre-injury level of sport was summarized as a percent with $95 \%$ confidence interval (CI). Statistical heterogeneity across pooled studies was assessed using the Tau $\left(\tau^{2}\right)$ and $\mathrm{I}^{2}$ statistic. An $\mathrm{I}^{2}$ value of $25 \%, 50 \%$ or $75 \%$ was considered a low, moderate or high level of heterogeneity respectively. ${ }^{18}$ The 'meta' package, version $4.9-2$, of the $\mathrm{R}$ statistical software package (version 3.3.1) was used for all statistical analyses (http://www.r-project.org/).

Study quality was assessed using two separate risk of bias tools, depending on study design. ${ }^{16}$ The Cochrane Risk of Bias Tool was used to assess RCTs. ${ }^{16}$ Each of the eight domains were marked as either low risk of bias (+), high risk of bias (-) or unclear risk of bias (?). ${ }^{16}$ Studies with the presence of three or more (-) or (?) were considered as having a high risk of bias. The Joanna Briggs Institute Checklist was used to assess the risk of bias in other study designs. ${ }^{19}$ Items were scored "yes (Y)", "no (N)", "unclear (U)" or "not applicable (N/A)" on the ten point checklist. ${ }^{19}$ If an individual study scored $\geqslant 6$ "yes $(\mathrm{Y})$ " scores, it was considered low risk of bias.
Completeness of reporting in intervention and control groups was assessed using relevant items of the Template for Intervention Description and Replication Checklist (TIDieR). ${ }^{20}$ Improved reporting provides an opportunity to understand the treatments used and more precisely inform clinicians about the type of interventions, enabling replication of treatments in clinical practice. If items were not relevant for an included study, they were scored not applicable (N/A). For example, completeness of reporting of a control group was scored N/A in studies where there was no control/comparison.

Both single group and comparative studies were assessed according to the GRADE guidelines. ${ }^{21}$ Overall quality was defined as high, moderate, low or very low. ${ }^{21}$ A staged process was followed whereby RCTs were first rated as high and case series were rated as low-quality evidence. ${ }^{22}$ Following this step, quality of evidence for individual outcomes were further reviewed, with the potential of being further downgraded by one level for each of the following factors: i) limitations in design ( $\geqslant 25 \%$ of the participants from studies with a high risk of bias as determined by the risk of bias tool), ii) inconsistency of results (significant statistical heterogeneity $\left(\mathrm{I}^{2}>40 \%\right)$ or inconsistent findings across studies ( $\leqslant 75 \%$ of the participants report findings in the same direction), iii) indirectness (i.e. generalization of the findings), iv) imprecision (total number of participants 
$<300$ for each outcome and wide confidence intervals) and v) other considerations (e.g. publication bias, flawed design, massive dropout). ${ }^{23,24}$ Single studies $(\mathrm{n}<300)$ were considered inconsistent and imprecise, providing "low quality evidence", which could be further downgraded to very lowquality evidence if additional items were not satisfied. ${ }^{23,24}$

\section{RESULTS}

The PRISMA flow diagram, is shown in Figure 1. 1924 studies were identified through database searching. Full texts of 34 studies were assessed for inclusion. Twelve studies met inclusion criteria.

Participant characteristics, study design and diagnostic criteria are displayed in Table 1. A total of 424 (229 males, 195 females) participants were included. Mean ages in individual studies ranged from 24 to 51 years. One-hundred and ninety-nine (47\%) participants were described as professional, competitive or high-level athletes, 75 (18\%) were recreational athletes and five participants were non-athletes. Activity levels in 145 (34\%) participants were not reported.

The majority of studies used clinical assessment and imaging to diagnose proximal hamstring tendinopathy (Table 1). The clinical assessments varied between studies. Imaging (magnetic resonance imaging or ultrasound) was used to attempt to verify proximal hamstring pathology in $92 \%$ of studies. A variety of patient reported physical function measures and pain measures were used. Mean followup times varied from one week to six years.

The most common intervention was platelet-rich plasma injection. ${ }^{25-29}$ Other interventions included surgery, $2,4,5,8$ corticosteroid injection, ${ }^{30,31}$ autologous whole blood injection, ${ }^{25}$ shockwave therapy ${ }^{1}$ and multi-modal intervention (NSAIDs, manual therapy, exercise and stretching). ${ }^{27,32}$

Name and description (item 1) and mode of delivery [item 6 (a) and (b)] were reported completely in both intervention and control groups (Figure 5). Intervention adherence (TIDieR items 11 and 12) was not reported in any study. No studies provided adequate details of post-surgical rehabilitation protocols. Risk of bias for RCTs and case series are reported in Figure 2 and Figure 3 respectively.

\section{EVIDENCE FROM RANDOMIZED CONTROLLED TRIALS}

There was very low-level evidence from a single RCT that shockwave therapy was more effective than a multi-modal intervention (one week of rest plus NSAIDs, two weeks of manual therapy and therapeutic ultrasound, and three weeks of exercise - including strength training and stretching) by a large effect in the short (-1.84 SMD; 95\% CI -2.59 , -1.09), medium (-3.23 SMD; 95\% CI -4.28, -2.19) and longterm $(-3.22$ SMD; 95\% CI $-4.28,-2.16)$ on self-reported symptoms (Table 2). ${ }^{1}$ There was also very low-level evidence that shockwave therapy was more effective than the multi-modal intervention by a large effect in the short-term (-3.09 SMD; 95\% CI -4.04, -2.15), medium (-2.90 SMD; 95\% CI -3.88, -1.92) and long-term (-2.42 SMD; 95\% CI-3.33, $-1.50)$ on physical function. ${ }^{1}$ Sixteen athletes $(80 \%)$ re-

\begin{tabular}{lcc}
\hline & $\begin{array}{c}\text { Cacchio } \\
\text { Davenport }\end{array}$ & $\begin{array}{c}\text { (2011) } \\
(2015)\end{array}$ \\
\hline $\begin{array}{l}\text { Random sequence generation (selection bias) } \\
\text { Allocation concealment (selection bias) }\end{array}$ & + & + \\
$\begin{array}{l}\text { Blinding of participants (performance bias) } \\
\text { Blinding of personnel (performance bias) } \\
\text { Blinding of outcome assessment (detection bias) }\end{array}$ & - & \\
$\begin{array}{l}\text { Incomplete outcome data (attrition bias) } \\
\text { Selective reporting (reporting bias) } \\
\text { Other sources of bias. }\end{array}$ & - & + \\
\hline Risk of bor & + & $?$ \\
\hline
\end{tabular}

Risk of bias for each domain for each RCT: - indicates high risk of bias, + indicates low risk of bias, ? indicates unclear risk of bias

\section{Figure 2: Risk of bias in RCTs}

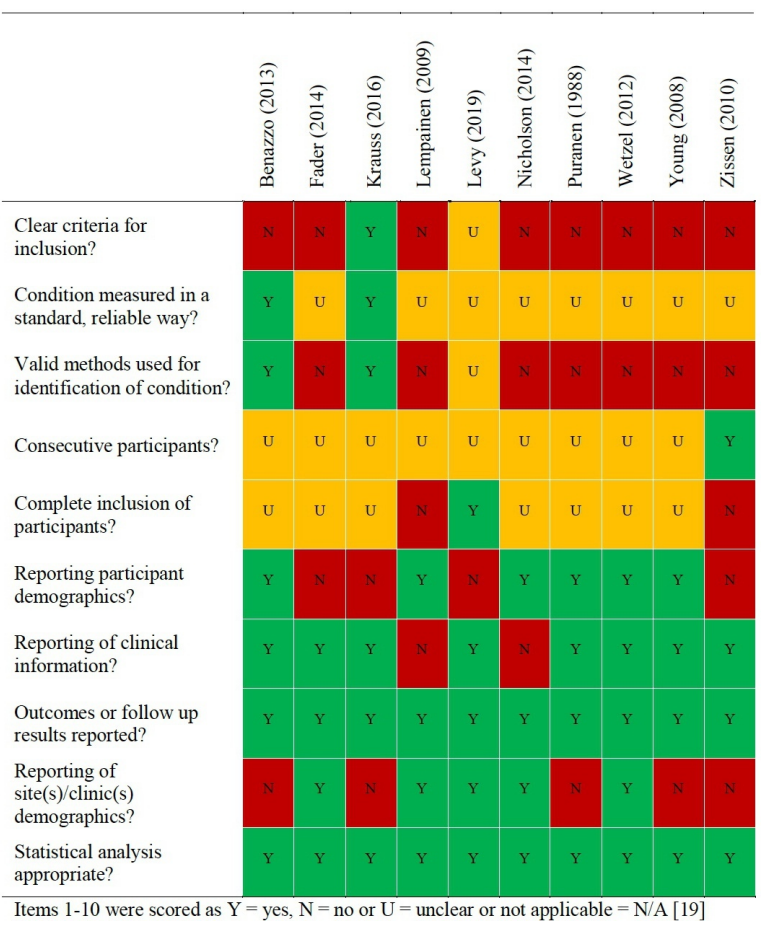

Figure 3: Risk of bias in case series

turned to pre-injury level of sport, at a mean time of nine weeks (range; 6-15 weeks) post shockwave therapy intervention (Figure 4 ). No participants in the multi-modal intervention group returned to sport at one year. ${ }^{1}$

There was very-low level evidence from a single RCT of no significant difference between platelet-rich plasma and autologous whole blood injection on physical function in the short [ $(0.03$ SMD; 95\% CI $-0.96,1.03)$ to $(0.28$ SMD 95\% CI -0.76, 1.32)] and medium-term [(0.17 SMD; 95\% CI -0.86, $1.21)$ to $(0.24$ SMD; $95 \%$ CI $-0.76,1.24)]$. There was also very low-level evidence of no difference between interventions on quality of life in the short (0.01 SMD; 95\% CI-1.00, 1.03) and medium-term (-0.04 SMD; 95\%CI -1.05, 0.97). ${ }^{25}$ One complication occurred after platelet-rich plasma injection (irritation of the sciatic nerve). ${ }^{25}$ 
Table 1: Patient Characteristics

\begin{tabular}{|c|c|c|c|c|c|}
\hline $\begin{array}{l}\text { Study } \\
\text { (Year) }\end{array}$ & $n(F)$, mean age (years) & Diagnostic criteria & $\begin{array}{l}\text { Tendon involved } \\
\text { (\%) }\end{array}$ & Activity level & $\begin{array}{l}\text { Average duration of } \\
\text { symptoms (range) }\end{array}$ \\
\hline \multicolumn{6}{|l|}{ RCTs } \\
\hline $\begin{array}{l}\text { Cacchio } \\
\text { (2011) }\end{array}$ & $\begin{array}{l}40 \text { ( } 13 F) \text {, SWT } 24 \text { years, multi-modal intervention } \\
24 \text { years }\end{array}$ & $\begin{array}{l}\text { At least } 2 \text { of the following painful; Puranen Orava test, fast } \\
\text { hamstring-stretch test, hamstring strength test } \\
\text { MRI }\end{array}$ & NR & $\begin{array}{l}\text { Multi-modal } \\
\text { professional } \\
\text { athletes: } 40\end{array}$ & $\begin{array}{l}\text { Multi-modal } \\
\text { intervention: } 21 \\
\text { months (13-81) } \\
\text { SWT: } 19.6 \text { months }\end{array}$ \\
\hline $\begin{array}{l}\text { Davenport } \\
\text { (2015) }\end{array}$ & $\begin{array}{l}15 \text { (13F), AWB: } 45.4 \text { years, PRP } 47 \text { years, } 17 \text { cases } \\
\text { ( } 2 \text { bilateral) }\end{array}$ & $\begin{array}{l}\text { Clinical diagnosis }{ }^{\mathrm{a}} \\
\text { Positive MRI or US }\end{array}$ & NR & NR & $N_{R}^{1-72)}$ \\
\hline \multicolumn{6}{|l|}{ Case series } \\
\hline $\begin{array}{l}\text { Benazzo } \\
(2013)\end{array}$ & $17(5 \mathrm{~F}), 27$ years & $\begin{array}{l}\text { Reporting pain at ischial tuberosity } \\
\text { Pain with concentric hamstring contraction: (positive } 47 \%) \\
\text { Tenderness ischial tuberosity ( } 41 \% \text { positive) } \\
\text { Puranen Orava test ( } 88 \% \text { positive) } \\
\text { Leg raising test ( } 23 \% \text { positive) } \\
\text { MRI }\end{array}$ & $\begin{array}{l}\text { Bicep femoris } \\
\text { (41\%) } \\
\text { Semimembranosus } \\
(29 \%) \\
\text { Semitendinosus } \\
(6 \%) \\
\text { Common tendon } \\
(23 \%)^{*}\end{array}$ & $\begin{array}{l}\text { Professional } \\
\text { athletes: } 9 \\
\text { Competitive } \\
\text { athletes: } 8\end{array}$ & 23 months (3-48) \\
\hline $\begin{array}{l}\text { Fader } \\
\text { (2014) }\end{array}$ & $18(12 \mathrm{~F}), 43$ years & $\begin{array}{l}\text { Clinical diagnosis } \\
\text { MRI }\end{array}$ & NR & NR & 32.6 months $(6-120)$ \\
\hline $\begin{array}{l}\text { Krauss } \\
(2016)\end{array}$ & 14 (13F), 47 years & $\begin{array}{l}\text { At least } 2 \text { of the following; } \\
\text { Tenderness to palpation at ischial tuberosity, positive bent } \\
\text { knee stretch test, positive supine plank test } \\
\text { MRI }\end{array}$ & NR & NR & $\begin{array}{l}4.1 \text { years ( } 5 \text { months } \\
\text { to } 10 \text { years) }\end{array}$ \\
\hline $\begin{array}{l}\text { Lempainen } \\
\text { (2009) }\end{array}$ & $\begin{array}{l}90(32 \mathrm{~F}), 34 \text { years, } \\
103 \text { cases }^{\mathrm{b}}(13 \text { bilateral) }\end{array}$ & $\begin{array}{l}\text { Patient interview and history } \\
\text { Pain at ischial tuberosity with hamstring stretch } \\
\text { MRI }\end{array}$ & $\begin{array}{l}\text { All } \\
\text { semimembranosus }\end{array}$ & $\begin{array}{l}\text { Professional: } 5 \\
\text { Competitive: } 47 \\
\text { Recreational: } 38\end{array}$ & NR \\
\hline $\begin{array}{l}\text { Levy } \\
\text { (2019) }\end{array}$ & $29(22 \mathrm{~F}), 45$ years & Clinical diagnosis $^{\mathrm{a}}$ confirmed with positive MRI & NR & NR & NR \\
\hline $\begin{array}{l}\text { Nicholson } \\
\text { (2014) }\end{array}$ & 18 (10F), 51 years, 22 cases ( 4 bilateral) & $\begin{array}{l}\text { Pain with prolonged sitting } \\
\text { Pain with hamstring contraction } \\
\text { MRI }\end{array}$ & NR & Athletes: 18 & 28 months $(2-120)$ \\
\hline $\begin{array}{l}\text { Puranen } \\
\text { (1988) }\end{array}$ & $\begin{array}{l}59(14 \mathrm{~F}) \text {, Athletes: } 25 \text { years, joggers: } 39 \text { years, non- } \\
\text { athletes: } 35 \text { years, } 65 \text { cases ( } 6 \text { bilateral) }\end{array}$ & $\begin{array}{l}\text { Pain on active stretching } \\
\text { Pain on palpation }\end{array}$ & NR & $\begin{array}{l}\text { Athletes: } 50 \\
\text { Joggers: } 4 \\
\text { Non-athletes: } 5\end{array}$ & NR \\
\hline $\begin{array}{l}\text { Wetzel } \\
(2012)\end{array}$ & 15 (8F), 38 years, 17 cases ( 2 bilateral) & $\begin{array}{l}\text { Clinical diagnosis } \\
\text { MRI }\end{array}$ & NR & $\begin{array}{l}\text { PRP group: } \\
\text { Collegiate or high- } \\
\text { level athletes: } 9 \\
\text { NR: } 1 \\
\text { Multi-modal } \\
\text { intervention group: } \\
\text { High level athletes: } \\
2 \\
\text { NR: } 3\end{array}$ & $\begin{array}{l}\text { Multi-modal } \\
\text { intervention: } 7.8 \\
\text { months } \\
\text { PRP: } 9.6 \text { months }\end{array}$ \\
\hline $\begin{array}{l}\text { Young } \\
\text { (2008) }\end{array}$ & 44 (16F), 29 years, 47 cases ( 3 bilateral) & $\begin{array}{l}\text { Pain on palpation of proximal hamstring region } \\
\text { Weakness at } 30^{\circ} \text { of resisted knee flexion } \\
\text { MRI/US }\end{array}$ & NR & $\begin{array}{l}\text { Professional: } 4 \\
\text { Semi-Professional: } \\
7 \\
\text { Recreational: } 33\end{array}$ & $\begin{array}{l}8 \text { cases: }<6 \text { months } \\
15 \text { cases: } 6-12 \\
\text { months } \\
10 \text { cases: } 12-18 \\
\text { months }\end{array}$ \\
\hline
\end{tabular}




\begin{tabular}{|c|c|c|c|c|c|}
\hline $\begin{array}{l}\text { Study } \\
\text { (Year) }\end{array}$ & $\mathrm{n}(\mathrm{F})$, mean age (years) & Diagnostic criteria & $\begin{array}{l}\text { Tendon involved } \\
\text { (\%) }\end{array}$ & Activity level & $\begin{array}{l}\text { Average duration of } \\
\text { symptoms (range) }\end{array}$ \\
\hline & & & & & $\begin{array}{l}14 \text { cases: }>18 \\
\text { months }\end{array}$ \\
\hline $\begin{array}{l}\text { Zissen } \\
(2010)\end{array}$ & 65 (37F), 37.7 years & $\begin{array}{l}\text { Clinical diagnosis }{ }^{\mathrm{a}} \\
\text { MRI/US }\end{array}$ & NR & NR & $\begin{array}{l}8 \text { cases: }<6 \text { months } \\
15 \text { cases: } 6 \text { months } \\
\text { to } 1 \text { year } \\
15 \text { cases: }>1 \text { year }\end{array}$ \\
\hline
\end{tabular}

$\mathrm{AWB}=$ autologous whole blood injection $\mathrm{F}=$ female, $\mathrm{MRI}=$ magnetic resonance imaging, $\mathrm{NR}=$ not reported, $\mathrm{PRP}=$ platelet-rich plasma injection, $\mathrm{SWT}=$ shockwave therapy, $\mathrm{US}=$ ultrasound 


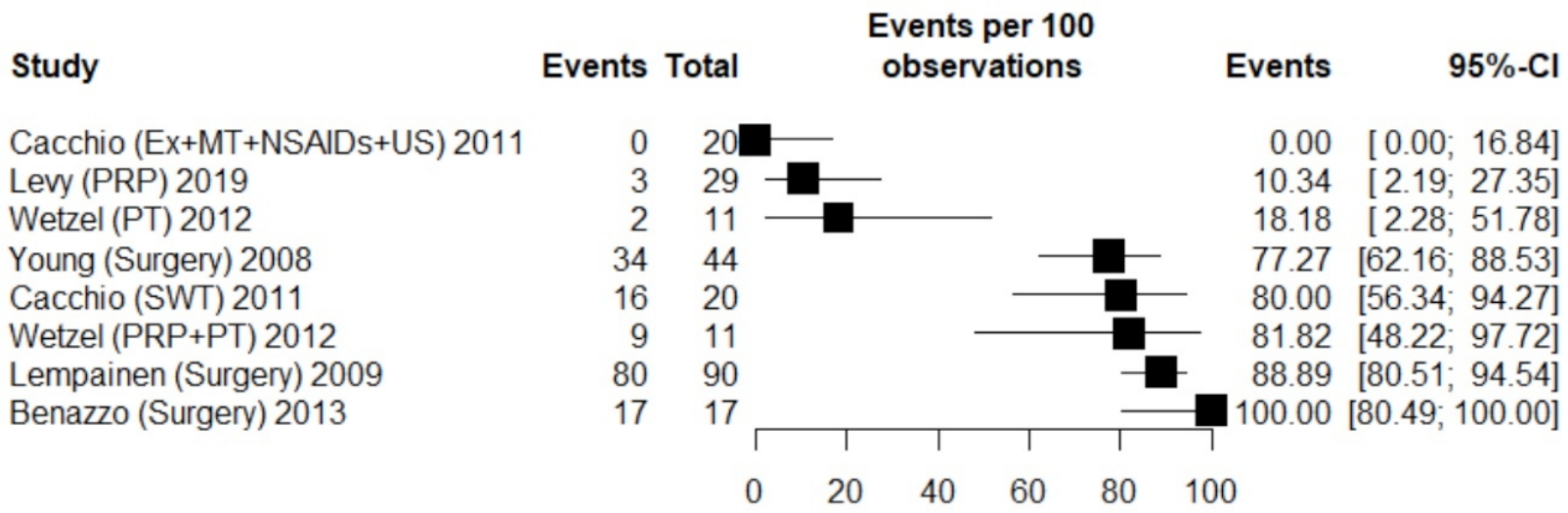

Demonstrates the proportion of participants returning to pre-injury level of sport in RCTs and case series

Ex: exercise, MT: manual therapy, PT: physical therapy, PRP: platelet-rich plasma injection, SWT: shockwave therapy, US: ultrasound

a Patient's who failed initial PT (physical therapy + NSAIDs) went on to receive a single volume PRP injection (6cc) plus additional physical therapy

Figure 4: Return to pre-injury level of sport
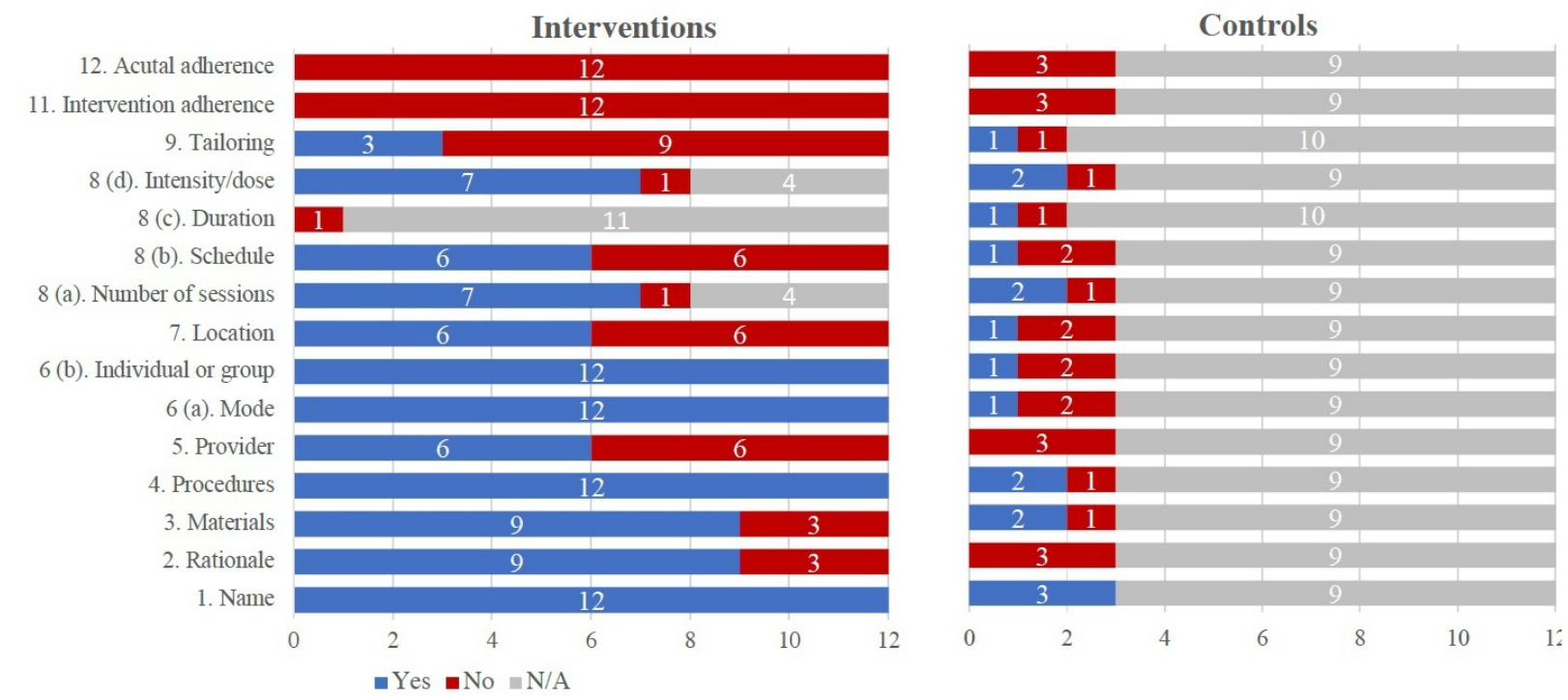

Percentage of trials achieving each TIDieR item in intervention and control groups of included studies (/12). Each item was scored as 'yes', 'not applicable 'or 'no' for both the intervention

and control groups in each included study.

Item 10 of the TIDiER scale was not reported on, as it was not able to be assessed in included studies.

In studies that reported on surgery or injection interventions item 11 and 12 were assessed on reporting on adherence to post-surgical protocol.

\section{Figure 5: Reporting of intervention and control group}

\section{EVIDENCE FROM CASE SERIES}

Three studies, recording the results of platelet-rich-plasma injection alone, documented change in self-reported symptoms over time (Table 2). 26,27,29 Outcomes could not be pooled due to heterogeneity in measures and incomplete data reporting. There was very low-level evidence that PRP is associated with a large reduction in symptoms over the short-term (-0.92 SPD; 95\% CI -1.54, -0.29). ${ }^{29}$ A second study found very low-level evidence that platelet-rich plasma injection was associated with a small, clinically significant, reduction in self-reported symptoms (Table 2). ${ }^{26}$
Two studies reported changes in physical function following platelet-rich plasma injection(s) over time (Table 2). ${ }^{28,29}$ There was very low-level evidence of a small $(-0.44$ SPD; $95 \%$ CI $-0.82,-0.06)^{28}$ to large $(-0.90$ SPD; $95 \%$ CI $-1.52,-0.28)^{29}$ improvement in physical function in the short-term (Table 2). Ten per cent of participants returned to their pre-injury level of sport after platelet-rich plasma injection at eight weeks (Figure 4$).{ }^{28}$ Two (4\%) minor adverse events occurred (short-term high levels of pain postinjection). ${ }^{26,28}$ 
Table 2: Results

\begin{tabular}{|c|c|c|c|c|c|}
\hline $\begin{array}{l}\text { Study } \\
\text { (Year) }\end{array}$ & Intervention & Mean follow up (range) & $\begin{array}{l}\text { Symptoms } \\
\text { Effect size (Cl) unless } \\
\text { otherwise stated } \\
\text { (-ve indicates reduction in } \\
\text { pain) }\end{array}$ & $\begin{array}{l}\text { Physical function } \\
\text { Mean effect size (CI) unless } \\
\text { otherwise stated }\end{array}$ & $\begin{array}{l}\text { Adverse effects } \\
\text { (number \%): minor, } \\
\text { major }\end{array}$ \\
\hline \multicolumn{6}{|l|}{ RCTs } \\
\hline $\begin{array}{l}\text { Cacchio } \\
\text { (2011) }\end{array}$ & $\begin{array}{l}\text { Multi-modal intervention: NSAIDs, manual therapy, } \\
\text { exercise and ultrasound } \\
\text { SWT: } 2500 \text { impulses at } 0.18 \mathrm{mJmm} 2 / \text { frequency = } 10 \\
\text { shocks in } 4 \text { separate sessions at weekly intervals. }\end{array}$ & 1 week, 6 months, 12 months & $\begin{array}{l}\text { Patient reported pain } \\
\text { rating: } \\
\text { VAS: } \\
\text { - Week 1: }-1.84(-2.59,-1.09) \\
\text { SMD } \\
\text { - } 6 \text { months: }-3.23(-4.28, \\
-2.19) \text { SMD } \\
\text { - } 12 \text { months: }-3.22(-4.28, \\
-2.16) \text { SMD } \\
\\
\text {-ve favours SWT }\end{array}$ & $\begin{array}{l}\text { Patient reported physical } \\
\text { function: } \\
\text { NPRS: } \\
\text { - } 1 \text { week: }-3.09(-4.04,-2.15) \text { SMD } \\
\text { - } 6 \text { months: }-2.90(-3.88,-1.92) \\
\text { SMD } \\
\text { - } 12 \text { months: }-2.42(-3.33,-1.50) \\
\quad \text { SMD } \\
\text {-ve favours SWT } \\
\text { Return to sport (pre-injury } \\
\text { level): } \\
\text { Proportion: } \\
\text { - SWT: } 16 / 20 \text { (80\%) mean time of } 9 \\
\quad \text { weeks (range, } 6-15 \text { weeks). } \\
\text { - Multi-modal intervention: } 0 / 20 \\
\text { (0\%) }\end{array}$ & $\begin{array}{l}\text { Minor: } \\
\text { - SWT: 0 (0\%) } \\
\text { Multi-modal interven- } \\
\text { tion: } 0(0 \%) \\
\\
\text { Major: } \\
\text { - SWT: 0 (0\%) } \\
\text { - Multi-modal interven- } \\
\text { tion: } 0(0 \%)\end{array}$ \\
\hline $\begin{array}{l}\text { Davenport } \\
\text { (2015) }\end{array}$ & $\begin{array}{l}\text { PRP vs AWB } \\
\text { Single U/S guided injection of AWB }(5 \mathrm{~mL}) \text { or PRP ( } 3 \mathrm{~mL}) \text {. }\end{array}$ & 12 weeks, 6 months & NR & 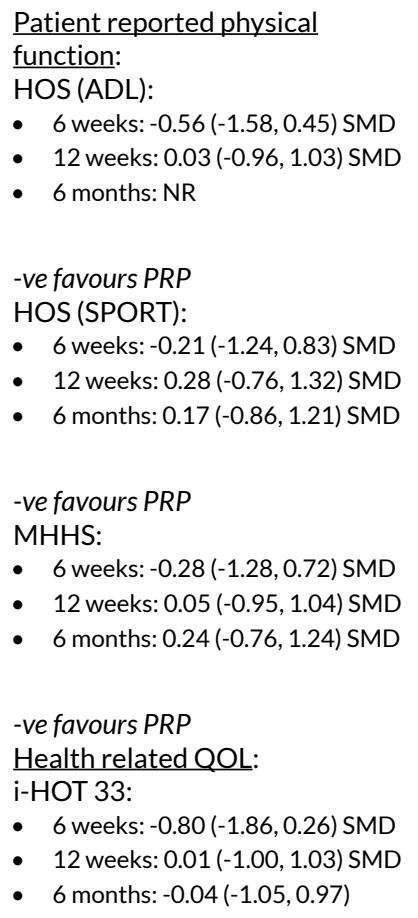 & $\begin{array}{l}\text { Minor: } \\
\text { - PRP: } 1(10 \%) \\
\text { - AWB: } 0(0 \%) \\
\text { Major: } \\
\text { PRP: } 0(0 \%) \\
\text { - AWB: } 0(0 \%)\end{array}$ \\
\hline
\end{tabular}




\begin{tabular}{|c|c|c|c|c|c|}
\hline $\begin{array}{l}\text { Study } \\
\text { (Year) }\end{array}$ & Intervention & Mean follow up (range) & $\begin{array}{l}\text { Symptoms } \\
\text { Effect size }(\mathrm{Cl}) \text { unless } \\
\text { otherwise stated } \\
\text { (-ve indicates reduction in } \\
\text { pain) }\end{array}$ & $\begin{array}{l}\text { Physical function } \\
\text { Mean effect size }(\mathrm{Cl}) \text { unless } \\
\text { otherwise stated }\end{array}$ & $\begin{array}{l}\text { Adverse effects } \\
\text { (number \%): minor, } \\
\text { major }\end{array}$ \\
\hline & & & & $\begin{array}{l}\text { SMD } \\
\text {-ve favours } P R P\end{array}$ & \\
\hline $\begin{array}{l}\text { Benazzo } \\
(2013)\end{array}$ & $\begin{array}{l}\text { Surgery } \\
\text { Prone incision from ischial tuberosity to } 8-15 \mathrm{~cm} \text { distally. } \\
\text { Partial transverse tenotomy performed plus sciatic nerve } \\
\text { release. }\end{array}$ & 71.3 months (24-138) & $\begin{array}{l}\text { Patient reported pain } \\
\text { rating: } \\
\text { VAS: } \\
\text { - }-6.02(-8.10,-3.94) S^{2} D^{a}\end{array}$ & 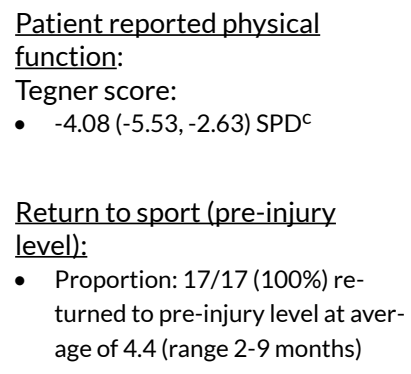 & $\begin{array}{l}\frac{\text { Minor: }}{1(6 \%)} \\
\frac{\text { Major: }}{1(6 \%)}\end{array}$ \\
\hline $\begin{array}{l}\text { Fader } \\
\text { (2014) }\end{array}$ & $\begin{array}{l}\text { PRP } \\
\text { A single U/S guided injection (2.5-4mL). }\end{array}$ & 46 months & 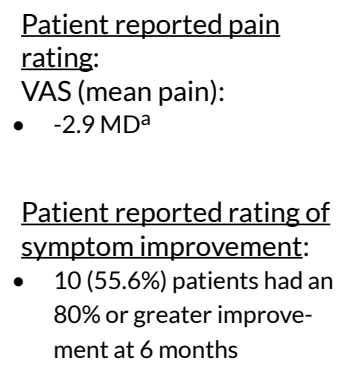 & NR & $\begin{array}{l}\text { Minor: } \\
\text { - } 1(6 \%) \\
\text { Major: } \\
\text { - } 0(0 \%)\end{array}$ \\
\hline $\begin{array}{l}\text { Krauss } \\
(2016)\end{array}$ & $\begin{array}{l}\text { PRP } \\
\text { Single U/S guided injection }(4 \mathrm{~mL})+\text { needle tenotomy }(5 \\
\text { passes). }\end{array}$ & 12 weeks & $\begin{array}{l}\text { Patient reported pain } \\
\text { rating: } \\
\text { VAS (mean pain): } \\
\text { - } \quad-0.92(-1.54,-0.29) \text { SPD }^{a}\end{array}$ & $\begin{array}{l}\text { Patient reported physical } \\
\text { function: } \\
\text { LEFS: } \\
\text { - } \quad-0.90(-1.52,-0.28) \text { SPD }^{c}\end{array}$ & $\begin{array}{l}\text { NR } \\
4(29 \%) \text { had worse } \\
\text { physical function at } \\
12 \text { weeks (LEFS) }\end{array}$ \\
\hline $\begin{array}{l}\text { Lempainen } \\
\text { (2009) }\end{array}$ & $\begin{array}{l}\text { Surgery } \\
\text { Prone, transverse or longitudinal incision. Transverse } \\
\text { tenotomy performed on semimembranosus + adhesions } \\
\text { freed around sciatic nerve as required. }\end{array}$ & $\begin{array}{l}49 \text { months (range, } 12-156 \\
\text { months) }\end{array}$ & NR & $\begin{array}{l}\text { Return to sport (pre-injury } \\
\text { level): } \\
\text { - Proportion: } 80 / 90 \text { (89\%) mean } 5 \\
\text { months }\end{array}$ & $\begin{array}{l}\text { Minor: } \\
\text { - } 3(3 \%) \\
\text { Major: } \\
\text { - } 1(1 \%)\end{array}$ \\
\hline \multirow[t]{2}{*}{$\begin{array}{l}\text { Levy } \\
\text { (2019) }\end{array}$} & $\begin{array}{l}\text { PRP } \\
\text { Single U/S guided injection }(6 \mathrm{~mL})\end{array}$ & 8 weeks & NR & $\begin{array}{l}\text { Patient reported physical } \\
\text { function: } \\
\text { - VISA H: }-0.44 \text { SPD }(-0.82,-0.06)\end{array}$ & Minor: \\
\hline & & & & $\begin{array}{l}\text { Return to Sport (pre-injury } \\
\text { level): }\end{array}$ & - $\frac{\text { Major: }}{0(0 \%)}$ \\
\hline
\end{tabular}




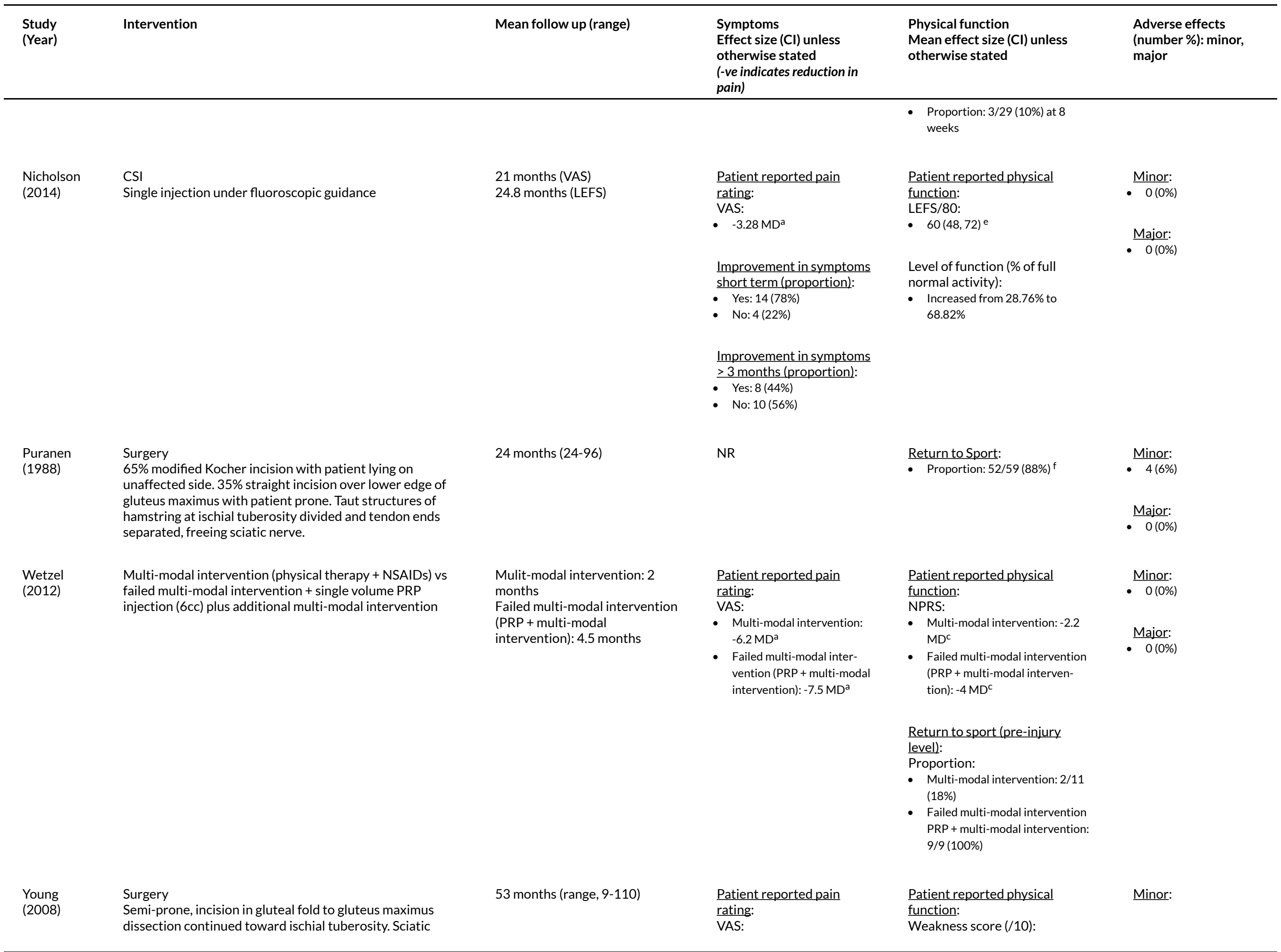




\begin{tabular}{|c|c|c|c|c|c|}
\hline $\begin{array}{l}\text { Study } \\
\text { (Year) }\end{array}$ & Intervention & Mean follow up (range) & $\begin{array}{l}\text { Symptoms } \\
\text { Effect size (CI) unless } \\
\text { otherwise stated } \\
\text { (-ve indicates reduction in } \\
\text { pain) }\end{array}$ & $\begin{array}{l}\text { Physical function } \\
\text { Mean effect size }(\mathrm{Cl}) \text { unless } \\
\text { otherwise stated }\end{array}$ & $\begin{array}{l}\text { Adverse effects } \\
\text { (number \%): minor, } \\
\text { major }\end{array}$ \\
\hline & nerve freed and prominent bursal tissue removed. & & - $-1.89(-2.36,-1.41) \mathrm{SPD}^{\mathrm{a}}$ & - $-3.69(-2.76,-4.62) \mathrm{MD}^{\mathrm{C}}$ & - $11(23 \%)$ \\
\hline \multirow[b]{2}{*}{$\begin{array}{l}\text { Zissen } \\
\text { (2010) }\end{array}$} & & \multirow[b]{2}{*}{48 months (6-96) } & & $\begin{array}{l}\text {-ve indicates reduction in } \\
\text { weakness Return to sport (pre- } \\
\text { injury level): } \\
\text { - Proportion: } 34 / 44(77 \%)\end{array}$ & Major: \\
\hline & Single CSI to area of maximum pain under $\mathrm{U} / \mathrm{S}$ guidance. & & $\begin{array}{l}\text { Patient reported rating: } \\
\text { Number (\%) of patient's } \\
\text { symptoms resolved: } \\
\text { - Complete: } 11(29 \%) \text {, mod- } \\
\text { erate: } 8(21 \%), \text { mild: } 10 \\
(26 \%), \text { no: } 9(24 \%)\end{array}$ & NR & $\begin{array}{l}\text { Minor: } \\
\text { - } 0(0 \%) \\
\text { Major: } \\
\text { - } 0(0 \%)\end{array}$ \\
\hline
\end{tabular}

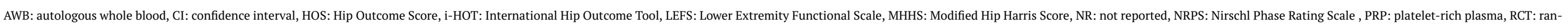

domised controlled trial, RTS: return to sport, SPD: standard paired difference, SMD: standard mean difference, SWT: shockwave therapy, U/S: ultrasound, VAS: Visual Analogue Scale, VISA H: Victorian Institute of Sport Assessment-proximal hamstring tendon
a Negative indicates an improvement in pain

a Negative indicates an improvement in pain
b Positive indicates improvement in weakness score

${ }^{\mathrm{c}}$ Negative indicates an improvement in physical function

d Adverse effects not reported on separately from complete tendon ruptures within study

e Measure taken post-operatively only

${ }^{\mathrm{f}}$ Level of return to sport not reported 
A single retrospective case series investigated the change in outcomes before and after PRP, in those who had failed a multi-modal intervention (physical therapy + non-steroidal anti-inflammatory drugs) ${ }^{27}$ Failure was determined by persistence of symptoms and an inability to return to baseline activity after the multi-modal intervention had been completed. ${ }^{27}$ There was very low-level evidence of an improvement in symptoms and physical function over time (Table 2). ${ }^{27}$ In this study, all athletes who were unable to return to sport with multi-modal intervention alone, returned to sport post PRP plus multi-modal intervention (Figure 4). ${ }^{27}$

Two studies reported change in self-reported symptoms after surgery (Table 2). ${ }^{2,8}$ There was very low-quality evidence of a large reduction in symptoms in the long-term [(-1.89 SPD; 95\% CI $-2.36,-1.41)$ to $(-6.02$ SPD; 95\% CI $-8.10,-3.94)]^{2,8}$ There was very low-quality evidence from a single study of a large improvement in physical function in the long-term (4.08 SPD; 95\%CI -5.53, -2.63) ..$^{2}$ Return to pre-injury level of sport following surgery ranged from $77-100 \%, 2,4,5,8$ with two studies reporting on mean return to sport time to pre-injury level (4.4 to 5 months). ${ }^{2,5}$ One study reported that $88 \%$ of participants returned to sport, but did not report whether participants returned to their pre-injury level. ${ }^{4}$

Adverse events were reported in all studies investigating outcomes of surgery (Table 2). 2,4,5,8 One study did not provide sufficient detail to allow events to be dichotomized as minor or major. ${ }^{4}$ Complications occurred in 21 (10\%) cases (Table 2). Type and severity of complications varied. Nineteen (9\%) patients experienced minor complications. Examples included: notable post-operative soreness ${ }^{5}$ and intermittent thigh paraesthesia that resolved spontaneously. ${ }^{2}$ Two (1\%) patients experienced major complications, which included wound abscess requiring surgical drainage ${ }^{2}$ and deep vein thrombosis. ${ }^{5}$

Management of proximal hamstring tendinopathy with corticosteroid injection was described in two studies. ${ }^{30,31}$ Both described change in self-reported symptoms over time (Table 1) ${ }^{30,31}$ One study provided very low-level evidence of clinically significant improvement in self-reported symptoms the long-term (Table 2). ${ }^{30}$ Both studies reported on long-term symptom resolution. 30,31 One study found that $56 \%$ of patients did not experience improvement greater than three months. ${ }^{30}$ The other study found that $56 \%$ still reported symptoms at long-term follow up. ${ }^{31} \mathrm{~A}$ single study reported on physical function (post-intervention only), as a percentage of full activity, and found activity level increased by $40 \%$ in the long-term (mean 24.8 months). ${ }^{30}$ No adverse events were reported. 30,31

\section{DISCUSSION}

The primary aim was to evaluate surgical and non-surgical interventions used to manage proximal hamstring tendinopathy. Three main findings emerged from the systematic review: i) there is a lack of rigorous RCTs comparing treatment interventions, ii) patient selection criteria and use of outcome measures are inconsistent across studies, and iii) there is inadequate description of treatment interventions used.

Of the twelve studies that met inclusion criteria, only two were RCTs. Both were confounded by small sample sizes. There was no high or moderate quality evidence for any intervention (symptoms, physical function or QOL). There was very low-level evidence from a single study to suggest that shockwave therapy was more effective than a multi-modal intervention (exercise, manual therapy, NSAIDs, ultrasound) in professional athletes, at both medium and long-term time points. ${ }^{1}$ While there were limitations in completeness of reporting on adverse events, surgery resulted in the highest level of minor and major adverse events.

Injection therapies may be an attractive option because they are less invasive than surgery. ${ }^{33}$ While multiple types of injections were reported (platelet-rich plasma, corticosteroid and autologous whole blood), the overall quality of evidence for all injections in proximal hamstring tendinopathy was found to be low or very low (Table 3). Consequently, at this stage it is not possible to recommend any type of injection over another or no injection.

Corticosteroid injections are a commonly prescribed treatment for tendinopathy. While it is important to consider the limitation in overall quality of evidence for corticosteroid injection in this review, the findings in a single group case series indicate a positive change in symptoms over time in the short-term (Table 2). Systematic reviews of high quality RCTs in tendinopathies, which compared corticosteroid injection to other interventions, found a similar trend of a positive short-term effect on symptoms, that are nullified in the medium and long-term. ${ }^{34,35}$ While this early improvement is desirable, it is worth noting that corticosteroid injection has been associated with delayed healing compared to wait and see (lateral epicondylagia) ${ }^{36,37}$ and increased collagen disorganization and necrosis in vitro. ${ }^{38}$

The popularity and cost of regenerative therapies warrants continued research to improve the evidence base. ${ }^{39}$ This systematic review found no high or moderate quality evidence for platelet-rich plasma injection; therefore, its utility remains uncertain in this condition. Its effect on symptoms in other tendinopathies was recently summarized in a systematic review. ${ }^{40}$ The authors reported that platelet-rich plasma injections were more efficacious than alternative injections on pain severity in tendinopathies (0.47, CI 95\% 0.22 to 0.72). ${ }^{40}$ However, it is worth noting that there were several limitations, including the type of injections used as comparisons (e.g. corticosteroid injection or saline) and the tendon involved. 


\begin{tabular}{|c|c|c|c|c|c|c|c|}
\hline No. of patients/studies & Limitations in Design & Inconsistency & Indirectness & Imprecision & Publication bias & Effect estimate & Certainty \\
\hline \multicolumn{8}{|l|}{ RCTs } \\
\hline \multicolumn{8}{|c|}{ SWT vs multi-modal intervention: VAS (follow up: 1 week; self-reported symptoms) } \\
\hline $40 / 1$ & Serious ${ }^{a}$ & Serious ${ }^{b}$ & Not serious & Serious $^{d}$ & Not serious ${ }^{\mathrm{e}}$ & Large (favours SWT) & $\bullet \circ \circ \circ$ VERY LOW \\
\hline \multicolumn{8}{|c|}{ SWT vs multi-modal intervention: VAS (follow up: 6 months; self-reported symptoms) } \\
\hline $40 / 1$ & Serious ${ }^{a}$ & Serious ${ }^{b}$ & Not serious & Serious $^{d}$ & Not serious ${ }^{\mathrm{e}}$ & Large (favours SWT) & $\bullet \circ \circ \circ$ VERY LOW \\
\hline \multicolumn{8}{|c|}{ SWT vs multi-modal intervention: VAS (follow up: 12 months; self-reported symptoms) } \\
\hline $40 / 1$ & Serious ${ }^{a}$ & Serious ${ }^{b}$ & Not serious & Serious $^{d}$ & Not serious $\mathrm{e}$ & Large (favours SWT) & $\bullet \bullet \circ \circ$ VERY LOW \\
\hline \multicolumn{8}{|c|}{ SWT vs multi-modal intervention: subjective rating of improvement (follow up: 1 week; self-reported symptoms) } \\
\hline $40 / 1$ & Serious ${ }^{\text {a }}$ & Serious ${ }^{b}$ & Not serious & Serious $^{d}$ & Not serious ${ }^{\mathrm{e}}$ & Large (favours SWT) & $\bullet \circ \circ \circ$ VERY LOW \\
\hline \multicolumn{8}{|c|}{ SWT vs multi-modal intervention: subjective rating of improvement (follow up: 6 months; self-reported symptoms) } \\
\hline $40 / 1$ & Serious ${ }^{a}$ & Serious ${ }^{b}$ & Not serious & Serious $^{d}$ & Not serious ${ }^{\mathrm{e}}$ & Large (favours SWT) & $\bullet \circ \circ \circ$ VERY LOW \\
\hline \multicolumn{8}{|c|}{ SWT vs multi-modal intervention: subjective rating of improvement (follow up: 12 months; self-reported symptoms) } \\
\hline $40 / 1$ & Serious ${ }^{a}$ & Serious $^{b}$ & Not serious & Serious $^{d}$ & Not serious ${ }^{\mathrm{e}}$ & Large (favours SWT) & $\bullet \circ \circ \circ$ VERY LOW \\
\hline \multicolumn{8}{|c|}{ SWT vs multi-modal intervention: NRPS (follow up: 1 week; physical function) } \\
\hline $40 / 1$ & Serious ${ }^{a}$ & Serious ${ }^{b}$ & Not serious & Serious $^{d}$ & Not serious ${ }^{\mathrm{e}}$ & Large (favours SWT) & $\bullet \bullet \circ \circ$ VERY LOW \\
\hline \multicolumn{8}{|c|}{ SWT vs multi-modal intervention: NRPS (follow up: 6 months; physical function) } \\
\hline $40 / 1$ & Serious ${ }^{a}$ & Serious $^{b}$ & Not serious & Serious $^{d}$ & Not serious ${ }^{\mathrm{e}}$ & Large (favours SWT) & $\bullet \bullet \circ \circ$ VERY LOW \\
\hline \multicolumn{8}{|c|}{ SWT vs multi-modal intervention: NRPS (follow up: 12 months; physical function) } \\
\hline $40 / 1$ & Serious $^{\text {a }}$ & Serious ${ }^{b}$ & Not serious & Serious $^{d}$ & Not serious ${ }^{\mathrm{e}}$ & Large (favours SWT) & $\bullet \circ \circ \circ$ VERY LOW \\
\hline \multicolumn{8}{|c|}{ SWT vs multi-modal intervention: return to pre-injury level of sport (follow up: 12 months; physical function) } \\
\hline $40 / 1$ & Serious $^{a}$ & Serious $^{b}$ & Not serious & Serious $^{d}$ & Not serious ${ }^{\mathrm{e}}$ & Large (favours SWT) & $\bullet \bullet \circ \circ$ VERY LOW \\
\hline \multicolumn{8}{|c|}{ SWT vs multi-modal intervention: adverse events (follow up: 12 months) } \\
\hline $40 / 1$ & Serious $^{\mathrm{a}}$ & Serious ${ }^{b}$ & Not serious & Serious $^{d}$ & Not serious ${ }^{\mathrm{e}}$ & Large (favours SWT) & $\bullet \circ \circ \circ$ VERY LOW \\
\hline \multicolumn{8}{|c|}{ PRP vs AWB: HOS ADL (follow up: mean 6 weeks; physical function) } \\
\hline $17 / 1$ & Serious ${ }^{a}$ & Serious ${ }^{b}$ & Not serious & Serious $^{d}$ & Not serious ${ }^{\mathrm{e}}$ & No apparent difference between groups & $\bullet \circ \circ \circ$ VERY LOW \\
\hline \multicolumn{8}{|c|}{ PRP vs AWB: HOS ADL (follow up: 12 weeks; physical function) } \\
\hline $17 / 1$ & Serious ${ }^{a}$ & Serious ${ }^{b}$ & Not serious & Serious $^{d}$ & Not serious ${ }^{\mathrm{e}}$ & No apparent difference between groups & $\bullet \circ \circ \circ$ VERY LOW \\
\hline \multicolumn{8}{|c|}{ PRP vs AWB: HOS Sport (follow up: 6 weeks; physical function) } \\
\hline $15 / 1$ & Serious $^{a}$ & Serious $^{b}$ & Not serious & Serious $^{d}$ & Not serious ${ }^{\mathrm{e}}$ & No apparent difference between groups & $\bullet \bullet \circ \circ$ VERY LOW \\
\hline \multicolumn{8}{|c|}{ PRP vs AWB: HOS Sport (follow up: 12 weeks; physical function) } \\
\hline $15 / 1$ & Serious $^{a}$ & Serious ${ }^{b}$ & Not serious & Serious $^{d}$ & Not serious ${ }^{\mathrm{e}}$ & No apparent difference between groups & $\bullet \bullet \circ \circ$ VERY LOW \\
\hline \multicolumn{8}{|c|}{ PRP vs AWB: HOS Sport (follow up: 6 months; physical function) } \\
\hline $15 / 1$ & Serious $^{\mathrm{a}}$ & Serious $^{b}$ & Not serious & Serious $^{d}$ & Not serious ${ }^{\mathrm{e}}$ & No apparent difference between groups & $\bullet \bullet \circ$ VERY LOW \\
\hline \multicolumn{8}{|c|}{ PRP vs AWB: iHOT 33 (follow up: 12 weeks; physical function) } \\
\hline $16 / 1$ & Serious ${ }^{a}$ & Serious $^{\mathrm{b}}$ & Not serious & Serious $^{d}$ & Not serious ${ }^{\mathrm{e}}$ & No apparent difference between groups & $\bullet \circ \circ \circ$ VERY LOW \\
\hline \multicolumn{8}{|c|}{ PRP vs AWB: iHOT 33 (follow up: 6 months; physical function) } \\
\hline $16 / 1$ & Serious $^{\mathrm{a}}$ & Serious $^{b}$ & Not serious & Serious $^{d}$ & Not serious ${ }^{\mathrm{e}}$ & No apparent difference between groups & $\bullet \bullet \circ \circ$ VERY LOW \\
\hline \multicolumn{8}{|c|}{ PRP vs AWB: MHHS (follow up: 12 weeks; physical function) } \\
\hline $17 / 1$ & Serious ${ }^{a}$ & Serious ${ }^{b}$ & Not serious & Serious $^{d}$ & Not serious ${ }^{\mathrm{e}}$ & No apparent difference between groups & • $০ \circ$ VERY LOW \\
\hline PRP vs AWB: MHHS (fol & nths; physical function) & & & & & & \\
\hline
\end{tabular}




\begin{tabular}{|c|c|c|c|c|c|c|c|}
\hline No. of patients/studies & Limitations in Design & Inconsistency & Indirectness & Imprecision & Publication bias & Effect estimate & Certainty \\
\hline $17 / 1$ & Serious $^{a}$ & Serious $^{b}$ & Not serious & Serious $^{d}$ & Not serious ${ }^{\mathrm{e}}$ & No apparent difference between groups & $\bullet \circ \circ \circ$ VERY LOW \\
\hline \multicolumn{8}{|c|}{ PRP vs AWB: adverse effects (follow up: 6 months) } \\
\hline $17 / 1$ & Serious ${ }^{a}$ & Serious ${ }^{b}$ & Not serious & Serious ${ }^{d}$ & Not serious ${ }^{e}$ & Not estimable & $\bullet \circ \circ \circ$ VERY LOW \\
\hline \multicolumn{8}{|l|}{ Case series } \\
\hline \multicolumn{8}{|c|}{ Surgery: VAS (follow up: 53-71 months; self-reported symptoms) } \\
\hline $61 / 2$ & Serious ${ }^{a}$ & Serious ${ }^{c}$ & Serious ${ }^{f}$ & Serious $^{d}$ & Not serious ${ }^{e}$ & Large & $\bullet \circ \circ \circ$ VERY LOW \\
\hline \multicolumn{8}{|c|}{ Surgery: Tegner Score (follow up: mean 71 months; physical function) } \\
\hline $17 / 1$ & Serious ${ }^{a}$ & Serious $^{b}$ & Serious ${ }^{f}$ & Serious $^{d}$ & Not serious ${ }^{\mathrm{e}}$ & Large & $\bullet \circ \circ \circ$ VERY LOW \\
\hline \multicolumn{8}{|c|}{ Surgery: subjective weakness score (follow up: mean 53 months; physical function) } \\
\hline $44 / 1$ & Serious ${ }^{a}$ & Serious ${ }^{b}$ & Serious ${ }^{f}$ & Serious ${ }^{d}$ & Not serious ${ }^{\mathrm{e}}$ & Not estimable & $\bullet \circ \circ \circ$ VERY LOW \\
\hline \multicolumn{8}{|c|}{ Surgery: return to pre-injury level of sport (follow up: 49-71 months; physical function) } \\
\hline $151 / 3$ & Serious ${ }^{\text {a }}$ & Serious $^{b}$ & Serious ${ }^{f}$ & Serious $^{d}$ & Not serious ${ }^{e}$ & Not estimable & $\bullet \circ \circ$ VERY LOW \\
\hline \multicolumn{8}{|c|}{ Surgery: return to undefined level of sport (follow up: mean 24 months; physical function) } \\
\hline $59 / 1$ & Serious ${ }^{\text {a }}$ & Serious ${ }^{b}$ & Serious ${ }^{f}$ & Serious ${ }^{d}$ & Not serious ${ }^{\mathrm{e}}$ & Not estimable & $\bullet \circ \circ \circ$ VERY LOW \\
\hline \multicolumn{8}{|c|}{ Surgery: adverse effects (follow up: $24-71$ months) } \\
\hline $179 / 4$ & Serious ${ }^{\text {a }}$ & Not serious & Serious ${ }^{f}$ & Serious $^{d}$ & Not serious ${ }^{\mathrm{e}}$ & Not estimable & $\bullet \circ \circ \circ$ VERY LOW \\
\hline \multicolumn{8}{|c|}{ Multi-modal intervention: vs multi-modal intervention: + delayed PRP + multi-modal intervention: NRPS (follow up: 4 and 4.5 months; physical function) } \\
\hline $17 / 1$ & Serious ${ }^{\text {a }}$ & Serious & Serious ${ }^{f}$ & Serious & Not serious & Not estimable & $\bullet \circ \circ \circ$ VERY LOW \\
\hline \multicolumn{8}{|c|}{ PRP: VISA H (follow up: 8 weeks; physical function) } \\
\hline $29 / 1$ & Serious ${ }^{\text {a }}$ & Serious ${ }^{b}$ & Serious ${ }^{f}$ & Serious $^{d}$ & Not serious ${ }^{e}$ & Small & $\bullet \circ \circ \circ$ VERY LOW \\
\hline \multicolumn{8}{|c|}{ PRP: LEFS (follow up: 12 weeks; physical function) } \\
\hline $14 / 1$ & Serious ${ }^{\text {a }}$ & Serious $^{b}$ & Serious ${ }^{f}$ & Serious ${ }^{d}$ & Not serious ${ }^{\mathrm{e}}$ & Large & $\bullet \circ \circ \circ$ VERY LOW \\
\hline \multicolumn{8}{|c|}{ PRP: adverse effects (follow up: 8 weeks- 46 months) } \\
\hline $47 / 2$ & Serious ${ }^{a}$ & Not serious & Serious ${ }^{f}$ & Serious $^{d}$ & Not serious ${ }^{e}$ & Not estimable & $\bullet \circ \circ \circ$ VERY LOW \\
\hline \multicolumn{8}{|c|}{ PRP: adverse effects (follow up: 12 weeks) } \\
\hline $14 / 1$ & Serious ${ }^{a}$ & Serious $^{b}$ & Serious $^{f}$ & Serious ${ }^{d}$ & Not serious ${ }^{\mathrm{e}}$ & Not estimable & $\bullet \circ \circ \circ$ VERY LOW \\
\hline \multicolumn{8}{|c|}{ CSI: VAS (assessed with: mean 21 months self-reported symptoms) } \\
\hline $18 / 1$ & Serious ${ }^{a}$ & Serious ${ }^{b}$ & Serious ${ }^{f}$ & Serious ${ }^{d}$ & Not serious ${ }^{e}$ & Not estimable & $\bullet \circ 0 \circ$ VERY LOW \\
\hline \multicolumn{8}{|c|}{ CSI: LEFS (follow up: mean 21 months; physical function) } \\
\hline $18 / 1$ & Serious ${ }^{a}$ & Serious $^{\mathrm{b}}$ & Serious $^{f}$ & Serious ${ }^{d}$ & Not serious ${ }^{\mathrm{e}}$ & Not estimable & $\bullet \bullet \circ \circ$ VERY LOW \\
\hline \multicolumn{8}{|c|}{ CSI: adverse effects (follow up: 21-48 months) } \\
\hline $83 / 2$ & Serious $^{a}$ & Not serious & Serious ${ }^{f}$ & Serious $^{d}$ & Not serious $\mathrm{e}$ & Not estimable & $\bullet \bullet \circ \circ$ VERY LOW \\
\hline
\end{tabular}

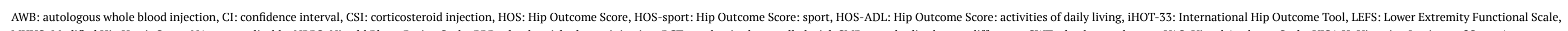

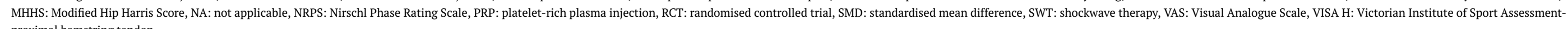
proximal hamstring tendon

$a \geqslant 25 \%$ of the participants from studies with a high risk of bia

b Single study $(\mathrm{n}<300)$

${ }^{\mathrm{c}} \mathrm{I}^{2}>40 \%$

d Pooled data with $<300$ participants for an outcome

e The possibility of publication bias is not excluded, but was not considered sufficient to downgrade the quality of evidence

${ }^{\mathrm{f}}$ No head-to-head comparison 
Shockwave therapy has been proposed for a host of upper and lower limb tendinopathies, 1,41-43,43,44,44,45 and other musculoskeletal conditions. ${ }^{46,47}$ This systematic review found preliminary support that shockwave therapy was superior to a multi-modal intervention (exercise, manual therapy, NSAIDs, ultrasound) for improving symptoms and physical function. However, as there was only a single study on shockwave therapy in proximal hamstring tendinopathy, with a small sample size, the results may not be representative of the wider population. ${ }^{1}$ Furthermore, there are several factors that may have made the comparison group (multi-modal intervention) less effective. In this study, exercise was included for three-weeks of the six-week program. In the literature, successful rehabilitation programs for other lower limb tendinopathies consistently report significantly longer time-frames. ${ }^{48-50}$ Avoiding excessive compressive load on the tendon at the enthesis has also been proposed to be a consideration in early to mid-stage exercise selection. ${ }^{6,51}$ In the multi-modal intervention (exercise, manual therapy, NSAIDs, ultrasound), exercises selected likely placed the insertion of the proximal hamstring tendon under high levels of compression, early in rehabilitation (lunge, hamstring stretch, exercise bike). ${ }^{1}$ For further information regarding a management program consistent with these parameters, readers are directed to a narrative review. $^{6}$

In managing tendinopathy, surgical interventions are typically reserved for recalcitrant cases that have not yet resolved with other interventions. While there were improvements over time following surgery (symptoms and physical function) in case series studies without comparator groups, it is unknown if these are real treatment effects or whether results are caused by other factors, such as natural history or postoperative rehabilitation. An insight into the likely treatment effects of surgery may be gleaned from a recent systematic review of upper and lower limb tendinopathy, which found surgery was not superior to sham surgery or physiotherapy on pain, function, range of motion and success ratings in the longer term. ${ }^{52}$ Recommendations were that surgery should not be seriously entertained until 12 months of an evidence based loading program has been credibly trialled. ${ }^{52}$ Until such time as there are adequately designed comparator studies, these recommendations should also be applied to proximal hamstring tendinopathy.

A limitation of this review was the lack of high-quality trials with consistent patient outcome measures, inclusion criteria and time points. The findings highlight the need for consensus on patient selection criteria, outcome measures and frequency of follow up, to allow the pooling of data. Another limitation was the high number of pre-post study designs. SPDs were calculated in an attempt to provide a standardized measure across study designs. It is important that these SPDs are not misconstrued as treatment effects, because there were no randomized comparisons that remove confounders such as regression to the mean, natural recovery and testing. Consequently, these studies were discussed separately from comparative designs. ${ }^{53}$ Interventions such as load management, heavy slow, strength training, platelet-rich plasma vs placebo and shockwave vs sham shockwave should be avenues for future research. Future research should prospectively report post-surgical protocols and adherence to interventions.

\section{CONCLUSION}

There was very low-level evidence that shockwave therapy was more effective than a multi-modal intervention. There was very low-level evidence of no difference between autologous whole blood injection and platelet-rich plasma injection. Results of this systematic review highlight the need for high-quality studies and the standardization of selection criteria, outcomes and reporting across studies. This will assist in determining the most effective option for management of proximal hamstring tendinopathy.

\section{ACKNOWLEDGMENTS}

The authors have no conflicts of interest to declare.

\section{SOURCE OF FUNDING}

none

Submitted: April 28, 2020 CST, Accepted: October 10, 2020 CST 


\section{REFERENCES}

1. Cacchio A, Rompe JD, Furia JP, Susi P, Santilli V, De Paulis F. Shockwave therapy for the treatment of chronic proximal hamstring tendinopathy in professional athletes. Am J Sports Med. 2011;39(1):146-153. doi:10.1177/0363546510379324

2. Benazzo F, Marullo M, Zanon G, Indino C, Pelillo F. Surgical management of chronic proximal hamstring tendinopathy in athletes: a 2 to 11 years of follow-up. J Orthopaed Traumatol. 2013;14(2):83-89. doi:10.100 7/s10195-013-0226-2

3. Lempainen L, Sarimo J, Mattila K, Orava S. Proximal hamstring tendinopathy-overview of the problem with emphasis on the surgical treatment. Oper Tech Sports Med. 2009;17(4):225-228. doi:10.105 3/j.otsm.2009.12.016

4. Puranen J, Orava S. The hamstring syndrome: a new diagnosis of gluteal sciatic pain. American Journal of Sports Medicine. 1988;16(5):517-521.

5. Lempainen L, Sarimo J, Mattila K, Vaittinen S, Orava S. Proximal hamstring tendinopathy: results of surgical management and histopathologic findings. Am J Sports Med. 2009;37(4):727-734. doi:10.1177/036 3546508330129

6. Goom TS, Malliaras P, Reiman MP, Purdam CR. Proximal hamstring tendinopathy: Clinical aspects of assessment and management. J Orthop Sports Phys Ther. 2016;46(6):483-493. doi:10.2519/jospt.2016.598 $\underline{6}$

7. Lempainen L, Johansson K, Banke IJ, et al. Expert opinion: diagnosis and treatment of proximal hamstring tendinopathy. Muscles Ligaments Tendons J. 2015;5(1):23-28.

8. Young IJ, van Riet RP, Bell SN. Surgical release for proximal hamstring syndrome. Am J Sports Med. 2008;36(12):2372-2378. doi:10.1177/03635465083229 $\underline{05}$

9. Liberati A, Altman DG, Tetzlaff J, et al. The PRISMA statement for reporting systematic reviews and meta-analyses of studies that evaluate healthcare interventions: explanation and elaboration. $B M J$. 2009;339:2700. doi:10.1136/bmj.b2700

10. van der Eng DM, Schepers T, Goslings JC, Schep NW. Rerupture rate after early weightbearing in operative versus conservative treatment of Achilles tendon ruptures: a meta-analysis. J Foot Ankle Surg. 2013;52(5):622-628. doi:10.1053/i.jfas.2013.03.027
11. White KE. High hamstring tendinopathy in 3 female long distance runners. J Chiropr Med. 2011;10(2):93-99. doi:10.1016/j.jcm.2010.10.005

12. Jayaseelan DJ, Moats N, Ricardo CR. Rehabilitation of proximal hamstring tendinopathy utilizing eccentric training, lumbopelvic stabilization, and trigger point dry needling: 2 case reports. $J$ Orthop Sports Phys Ther. 2014;44(3):198-205. doi:10.2 519/jospt.2014.4905

13. Jacobson JA, Rubin J, Yablon CM, Kim SM, Kalume-Brigido M, Parameswaran A. UltrasoundGuided Fenestration of Tendons About the Hip and Pelvis: Clinical Outcomes. J Ultrasound Med. 2015;34(11):2029-2035. doi:10.7863/ultra.15.01009

14. Lawrenson PR, Crossley KM, Vicenzino BT, et al. Muscle size and composition in people with articular hip pathology: a systematic review with metaanalysis. Osteoarthritis Cartilage. 2019;27(2):181-195. doi:10.1016/j.joca.2018.10.008

15. Borenstein M, Hedges LV, Higgins JPT, Rothstein H. Introduction to Meta-Analysis.; 2011.

16. Higgins JPT, Green S, eds. Cochrane Handbook for Systematic Reviews of Interventions Version 5.1.0 [Updated March 2011]. The Cochrane Collaboration; 2011.

17. Cohen J. Statistical Power Analysis for the Behavioral Sciences. Hillsdale, N.J.: L. Erlbaum Associates; 1988.

18. Higgins JP, Thompson SG, Deeks JJ, Altman DG. Measuring inconsistency in meta-analyses. BMJ. 2003;327(7414):557-560. doi:10.1136/bmj.327.7414.5 $\underline{57}$

19. Aromataris E, Munn Z, eds. Joanna Briggs Institute Reviewer's Manual. The Joanna Briggs Institute; 2017. https://joannabriggs.org/critical-appraisal-tools.

20. Hoffmann TC, Glasziou PP, Boutron I, et al. Better reporting of interventions: template for intervention description and replication (TIDieR) checklist and guide. BMJ. 2014;348:1687. doi:10.1136/bmi.g1687

21. Balshem H, Helfand M, Schunemann HJ, et al. GRADE guidelines: 3. Rating the quality of evidence. J Clin Epidemiol. 2011;64(4):401-406. doi:10.1016/i.jcli nepi.2010.07.015

22. Atkins D, Best D, Briss PA, et al. Grading quality of evidence and strength of recommendations. $B M J$. 2004;328(7454):1490. doi:10.1136/bmj.328.7454.1490 
23. Guyatt GH, Oxman AD, Vist GE, et al. GRADE: an emerging consensus on rating quality of evidence and strength of recommendations. BMJ. 2008;336(7650):924-926. doi:10.1136/bmj.39489.4703 47.AD

24. Thoomes EJ, Scholten-Peeters W, Koes B, Falla D, Verhagen AP. The effectiveness of conservative treatment for patients with cervical radiculopathy: a systematic review. Clin J Pain. 2013;29(12):1073-1086. doi:10.1097/AJP.0b013e31828441fb

25. Davenport KL, Campos JS, Nguyen J, Saboeiro G, Adler RS, Moley PJ. Ultrasound guided intratendinous injections with platelet-rich plasma or autologous whole blood for treatment of proximal hamstring tendinopathy: a double-blind randomized controlled trial. J Ultrasound Med. 2015;34(8):1455-1463. doi:1 $\underline{0.7863 / \text { ultra.34.8.1455 }}$

26. Fader RR, Mitchell JJ, Traub S, et al. Platelet-rich plasma treatment improves outcomes for chronic proximal hamstring injuries in an athletic population. Muscles Ligaments Tendons J. 2014;4(4):461-466.

27. Wetzel RJ, Patel RM, Terry MA. Platelet-rich plasma as an effective treatment for proximal hamstring injuries. Orthopedics. 2013;36(1):64-70. do i:10.3928/01477447-20121217-20

28. Levy GM, Lucas P, Hope N. Efficacy of a plateletrich plasma injection for the treatment of proximal hamstring tendinopathy: A pilot study. J Sci Med Sport. 2019;22(3):247-252. doi:10.1016/j.jsams.2018.0 8.001

29. Krauss J, Bodor M, Nugent R, Fredericson M. Platelet rich plasma injections in high hamstring tendinopathy. PM and R. 2014;6(9):S276.

30. Nicholson LT, DiSegna S, Newman JS, Miller SL. Fluoroscopically guided peritendinous corticosteroid injection for proximal hamstring tendinopathy: a retrospective review. Orthop J Sports Med. 2014;2(3):2325967114526135. doi:10.1177/232596711 $\underline{4526135}$

31. Zissen MH, Wallace G, Stevens KJ, Fredericson M, Beaulieu CF. High hamstring tendinopathy: MRI and ultrasound imaging and therapeutic efficacy of percutaneous corticosteroid injection. $\mathrm{Am} \mathrm{J}$ Roentgentology. 2010;195(4):993-998. doi:10.2214/AJ R.09.3674

32. Cacchio A, De Paulis F, Maffulli N. Development and validation of a new visa questionnaire (VISA-H) for patients with proximal hamstring tendinopathy. Br J Sports Med. 2014;48(6):448-452. doi:10.1136/bjsp orts-2012-091552
33. Orchard JW, Saw R, Masci L. The use of ultrasound-guided injections for tendinopathies. Sports Imaging. 2018;6(10):38. doi:10.1007/s40134-01 8-0296-2

34. Coombes BK, Bisset L, Vicenzino B. Efficacy and safety of corticosteroid injections and other injections for management of tendinopathy: a systematic review of randomised controlled trials. Lancet.

2010;376(9754):1751-1767. doi:10.1016/s0140-6736(1 0)61160-9

35. Cook T, Minns Lowe C, Maybury M, Lewis JS. Are corticosteroid injections more beneficial than anaesthetic injections alone in the management of rotator cuff-related shoulder pain? A systematic review. Br J Sports Med. 2018;52(8):497-504. doi:10.11 36/bjsports-2016-097444

36. Bisset L, Beller E, Jull G, Brooks P, Darnell R, Vicenzino B. Mobilisation with movement and exercise, corticosteroid injection, or wait and see for tennis elbow: randomised trial. BMJ (Clinical research ed). 2006;333(7575):939-939. doi:10.1136/bmj.3896 1.584653. AE

37. Smidt N, van der Windt DA, Assendelft WJ, Deville WL, Korthals-de Bos IB, Bouter LM. Corticosteroid injections, physiotherapy, or a wait-and-see policy for lateral epicondylitis: a randomised controlled trial. Lancet. 2002;359(9307):657-662. doi:10.1016/s0140-6 736(02)07811-x

38. Dean BJ, Lostis E, Oakley T, Rombach I, Morrey ME, Carr AJ. The risks and benefits of glucocorticoid treatment for tendinopathy: a systematic review of the effects of local glucocorticoid on tendon. Semin Arthritis Rheum. 2014;43(4):570-576. doi:10.1016/i.se marthrit.2013.08.006

39. Zhang JY, Fabricant PD, Ishmael CR, Wang JC, Petrigliano FA, Jones KJ. Utilization of platelet-rich plasma for musculoskeletal Injuries: an analysis of current treatment trends in the United States. Orthop J Sports Med. 2016;4(12):1-7. doi:10.1177/2325967116 $\underline{676241}$

40. Miller LE, Parrish WR, Roides B, Bhattacharyya S. Efficacy of platelet-rich plasma injections for symptomatic tendinopathy: systematic review and meta-analysis of randomised injection-controlled trials. BMJ Open Sport Exer Med. 2017;3(1):000237. do i:10.1136/bmjsem-2017-000237

41. Furia JP, Rompe JD, Cacchio A, Del Buono A, Maffulli N. A single application of low-energy radial extracorporeal shock wave therapy is effective for the management of chronic patellar tendinopathy. Knee Surg Sports $\operatorname{Tr}$ A. 2013;21(2):346-350. doi:10.1007/s00 $\underline{167-012-2057-8}$ 
42. Rompe JD, Segal NA, Cacchio A, Furia JP, Morral A, Maffulli N. Home training, local corticosteroid injection, or radial shock wave therapy for greater trochanter pain syndrome. Am J Sports Med. 2009;37(10):1981-1990. doi:10.1177/03635465093343 $\underline{74}$

43. Rompe JD, Furia J, Maffulli N. Eccentric loading compared with shock wave treatment for chronic insertional achilles tendinopathy. A randomized, controlled trial. J Bone Jt Surg. 2008;90(1):52-61. doi:1 $\underline{0.2106 / \text { ibjs.F.01494 }}$

44. Rompe JD, Furia J, Maffulli N. Eccentric loading versus eccentric loading plus shock-wave treatment for midportion achilles tendinopathy: a randomized controlled trial. Am J Sports Med. 2009;37(3):463-470. doi:10.1177/0363546508326983

45. Furia JP, Rompe JD, Maffulli N. Low-energy extracorporeal shock wave therapy as a treatment for greater trochanteric pain syndrome. Am J Sports Med. 2009;37(9):1806-1813. doi:10.1177/036354650933301 4

46. Rompe JD, Cacchio A, Furia JP, Maffulli N. Lowenergy extracorporeal shock wave therapy as a treatment for medial tibial stress syndrome. Am J Sports Med. 2010;38(1):125-132. doi:10.1177/0363546 $\underline{509343804}$

47. Li W, Pan Y, Yang Q, Guo Z-G, Yue Q, Meng Q-G. Extracorporeal shockwave therapy for the treatment of knee osteoarthritis: A retrospective study. Medicine. 2018;97(27):11418-11418. doi:10.1097/M D.0000000000011418
48. Bisset L, Beller E, Jull G, Brooks P, Darnell R, Vicenzino B. Mobilisation with movement and exercise, corticosteroid injection, or wait and see for tennis elbow: randomised trial. BMJ. 2006;333(7575):939. doi:10.1136/bmj.38961.584653.A E

49. Kongsgaard M, Kovanen V, Aagaard P, et al. Corticosteroid injections, eccentric decline squat training and heavy slow resistance training in patellar tendinopathy. Scand J Med Sci Sports. 2009;19(6):790-802. doi:10.1111/j.1600-0838.2009.00 949.x

50. Mellor R, Bennell K, Grimaldi A, et al. Education plus exercise versus corticosteroid injection use versus a wait and see approach on global outcome and pain from gluteal tendinopathy: prospective, single blinded, randomised clinical trial. BMJ (Clinical research ed). 2018;361:1662-1662. doi:10.1136/bmj.k1 662

51. Cook JL, Purdam C. Is compressive load a factor in the development of tendinopathy? Br J Sports Med. 2012;46(3):163-168. doi:10.1136/bjsports-2011-09041 $\underline{4}$

52. Challoumas D, Clifford C, Kirwan P, Millar NL. How does surgery compare to sham surgery or physiotherapy as a treatment for tendinopathy? A systematic review of randomised trials. BMJ Open Sport Exercise Medicine. 2019;5(1):e000528. doi:10.11 36/bmjsem-2019-000528

53. Herbert R. Practical Evidence-Based Physiotherapy. Edinburgh: Elsevier, Churchill Livingstone; 2015. 


\section{APPENDIX}

Table 4: Medline Search

\begin{tabular}{lll}
\hline $\begin{array}{l}\text { Date } \\
\text { Set }\end{array}$ & $\begin{array}{l}30.01 .2019 \\
\text { Results }\end{array}$ \\
\# 3 & 658 & $\begin{array}{l}\text { \#2 AND \#1 } \\
\text { Indexes=MEDLINE Timespan=All years } \\
\text { (((TOPIC: (tendinopathy) OR TOPIC: (tendinitis)) OR TOPIC: (tendinosis)) OR TOPIC: (tear)) } \\
\text { Indexes=MEDLINE Timespan=All years } \\
\text { (((TOPIC: (hamstring) OR TOPIC: (biceps } \\
\text { femoris)) OR TOPIC: (semitendinos*)) OR TOPIC: (semimembranos*)) } \\
\text { Indexes=MEDLINE Timespan=All years }\end{array}$ \\
\hline
\end{tabular}

